\title{
Easy Implementation of Intra-period Water Market Clearing Using CDDP Models
}

\author{
I. Mahakalanda
}

\begin{abstract}
This paper shows how to clear a market for water, spatially with both consumptive and non-consumptive uses of water. A set of demand/supply nodes in a tree, radiating from a single reservoir represents a mixed-use river catchment. The multi-nodal version of the deterministic Constructive Dual Dynamic Programming (CDDP) procedure developed in this study constructs a net demand curve for water released for the reservoir which to efficiently and implicitly clears the market across all nodes in the catchment. The main advantage of the CDDP approach is that it enables us to backtrack and pick apart prices in ways that allow participants and analysts to understand why prices are, what they are, and from where the value derives.
\end{abstract}

Index Terms-Water markets, CDDP, consumptive use, non-consumptive use.

\section{INTRODUCTION}

While water markets are often regarded as the most promising method of managing this increasingly stressed natural resource, the literature on water market concepts is still emerging. Our work involves developing a set of market mechanisms to support trading of storable surface water in such multi-use catchments. We can identify two distinct categories of water "user", or beneficiary, in any particular sub-system. "Consumptive users" benefit from "extracting" water. The water system does not measurably receive the consumptive use over which the market operates. But "non-consumptive users" benefit from the flow of water between two points in the water system being at certain levels, without extracting any measurable quantity at all. Such benefits may come from the hydro generation, but also from recreation, navigation, flood control or environmental effects.

The market would receive some offers from irrigators, municipalities, wanting to extract water for "consumptive use." On the other hand, hydro generators create non-consumptive demands who require water flow in particular channels for power generation purposes. In reality, though, environmental priorities such as minimum instream levels might be expressed by flow and storage limits. But, under a market setting, offers could generate from agencies wanting to see either higher or lower water flows in particular channels to improve environmental, recreational, navigational or flood control objectives. Although we have not modeled it, the same would be applicable for flows over time, in the form of reservoir storage. Adding constraints to

Manuscript received January 31, 2018; revised May 29, 2018.

I. Mahakalanda is with Department of Decision Sciences, University of Moratuwa, Sri Lanka (e-mail: indra.mahakalanda@gmail.com). reflect physical and regulatory limits on water flows and levels, produces a constrained value maximisation formulation similar to that employed in electricity markets, and solving that optimisation produces both optimal dispatches and prices, for water. For example, see [1] and [2].

Mahakalanda in [3] has developed a nodal catchment model and its easy implementation procedure. This nodal Constructive Dual Dynamic Programming (CDDP) procedure can be applied to clear an intra-temporal market involving both consumptive and non-consumptive users in any catchment with one long-term reservoir and a tree configuration. And Starkey et al [1] and Starkey [2] developed a two-level application of CDDP to perform inter-temporal market-clearing in that context. A stochastic CDDP constructs aggregate demand curves for storage in the single reservoir for each period. First, though, a multi-nodal deterministic CDDP constructs aggregate demand curves for water at each node, and ultimately for reservoir release, in each period. Those demand curves integrate the impact of consumptive and non-consumptive bids on various arcs and nodes, working inwards from the "leaves" of the catchment tree to the "root," at the main reservoir. The complexity of the intra-period network can readily be increased by adding upstream and downstream nodes, and physical links, while still maintaining efficient computation. Increasing the number of long term storage reservoirs will increase the complexity of the procedure, though, because the "curse of dimensionality" will eventually apply to any DP-based technique for multiple reservoirs.

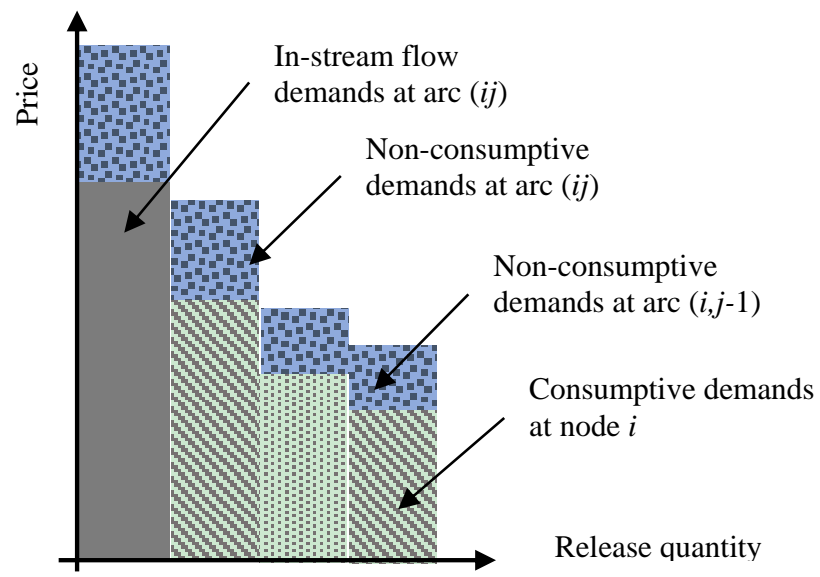

Fig. 1. Demand curve for water release.

Thus, a Linear Programming (LP) based market clearing model could provide greater computational efficiency for actual market clearing, particularly for multi-reservoir catchments [2]. But the CDDP approach allows us to backtrack and pick apart prices in ways that provide participants and analysts to understand why prices are what 
they are, and where the value is being derived from, as in Fig. 1. The marginal value (or price) of water at any point in the network, and particularly the marginal value of water stored for future use in the reservoir, thus represents the interplay between all potential uses in the catchment, both consumptive and non-consumptive, both upstream and downstream from the reservoir.

This paper is organised in the following ways: Section II briefly reviews relevant past work in the area of wholesale markets for tradable surface water. Section III models the multi-period deterministic nodal water market clearing using LP. Section IV develops intra-period nodal CDDP procedure. Section $\mathrm{V}$ is an illustrative example followed by the conclusions in Section VI.

\section{REVIEW OF PREVIOUS WORKS}

In an integrated catchment market, for a single period, participants are assumed to submit bids reflecting the value they place on the consumptive use of water at nodes, e.g. for agriculture or municipal water supply and/or for the non-consumptive use of water flowing between nodes, principally for power generation, but potentially for environmental or recreational uses, too. There may also be suppliers offering water, or pumping services. Such an intra-period market could be cleared using an LP model, such as those used to clear electricity markets. The literature on LP based surface water network market modelling starts with [4] Murphy et al. [5] illustrated how to achieve an efficient water allocation using auction based mechanisms. More recent literature discusses a number of water market issues such as locational marginal prices, revenue adequacy [6] and in-stream flow values.

But this paper is based on the works of [3] and this demonstrates a Constructive Dual Dynamic Programming (CDDP) model that can be used to construct aggregate demand curves for water at each node in the network, in that period. The market solution is then determined by the intersection of the demand curve for reservoir release, with the supply curve determined by inter-period market-clearing.

[1] and [2] describe experiments using CDDP to determine the value that could be added by using stochastic optimization in an inter-temporal market clearing context. However, those previous studies (see [1], [6]-[8]) do not explicitly address the structure of the river network, and interactions between consumptive and non-consumptive water users at various points within it, whether by a market or otherwise. The benefit from immediate release is modelled by a "Demand Curve for Release" $(d c r)$, in each period. This paper describes an efficient intra-period CDDP procedure to determine the $d c r$ s from a river network. The intra-period optimizations are deterministic, forming sub-problems to an overall stochastic model.

River networks have a number of features affecting the demand for reservoir release. A stream or inflow that flows into the catchment is known as a tributary flow. These tributary flows are often subject to temporal variations. The river flow is often diverted to cater for ecological demands and consumptive uses; these diversions typically have only a remote chance to return to the catchment directly. Non-consumptive uses, on the other hand, gain benefit (or in the case of pumps, incur costs) based on water flow. The water itself is still available for further use downstream. Both contribute to defining a $d c r$.

The $d c r$ defines the amount of water the reservoir manager should economically release, given the benefit from supplying water to consumptive and non-consumptive users, as a function of the marginal value of stored water which could be determined using benefit curves estimated by the reservoir manager, but in this application we envisage the benefits being defined by clearing a market within which each consumptive user bids for water to be delivered to their node, and each non-consumptive user bids for water flow on their arc. To clear the full inter-temporal market requires the determination of a $d c r$ for every inflow state in every period over the planning horizon.

\section{Multi-PERIOD Deterministic NodAl (PRIMAL) LP MODEL FOR WATER MARKETS}

\section{A. Model}

A simple deterministic version of the inter-period model that uses the benefit function from a nodal optimization is illustrated in the following. For the interest of simplicity, let us assume an operating policy where the market aims to maximize the benefit of allocating water in each period over the planning horizon based on the available inflows to the reservoir. Here, $T$ is the number of periods, $s_{t}$ is the reservoir storage for period $t, \bar{s}$ the storage capacity, $r_{t}$ is the net release (accounting for inflow) which implicitly represents the met demand in a particular time period, and $s_{0}$ represents the initial and target storage level. $z_{t}$ is the spill (unusable overflow) during the period. The bounds on $r_{t}$ could be implicit in the definition of $N B_{t}$.

A general inter-period benefit maximizing objective can be written by considering both intra-period benefit function, $N B(r)$ and the future benefit function, $V_{T+1}\left(s_{T+1}\right)$ as follows:

$$
\max _{r, s}\left(\sum_{t=1}^{T} N B_{t}\left(r_{t}\right)+V_{T+1}\left(s_{T+1}\right)\right)
$$

Subject to:

$$
\begin{gathered}
s_{t+1}=s_{t}-r_{t}-z_{t}, \quad \forall t=1, \ldots, T \\
\underline{R} \leq r_{t} \leq \bar{R}, \quad \forall t=1, \ldots, T \\
s_{1}=s_{0}, s_{T+1}=s_{0} \\
0 \leq s_{t} \leq \bar{s}, \quad \forall t=1, \ldots, T
\end{gathered}
$$

\section{B. Explanation}

The objective function (1) seeks to clear the water market by maximizing the release benefits, plus future storage benefits, assuming that the bids represent the true economic contribution of the water used by activities across the network.

Constraint (2) ensures continuity of storage balance across time $^{1}$. In this model $r$ can take negative values (net flow

\footnotetext{
${ }^{1}$ When the net increase in storage, $\left(s_{t+1}-s_{t}\right)$, is less than the net inflow, $-r_{t}$, the system operator has been forced to spill some inflow coming in the time period $t$ as the intra-period model is unable to make use of it all.
} 
injections) as the net release includes both inflows and releases at the reservoir node.

Constraint (3) shows feasibility bounds for the net release. Here for the interest of simplicity we include a spill variable $z_{t}$, with relevant bounds. Instead a separate spill node could be included in the intra-period model to take care any spill from the reservoir. $z_{t}$ is non-zero only when the end-of-period storage is at reservoir capacity or the target storage in the last period.

Constraint (4) maintains initial and end of the final horizon (target) storage value. The restriction, $s_{T+1}=s_{0}$ implies a steady state condition. Instead, the end-of-horizon storage could be left free by including an end-of-horizon benefit function, for case of a single year operation.

Constraint (5) provides the lower and upper storage bounds.

In a typical Stochastic LP (SLP) formulation, the intra-period optimization sub-problem is embedded within the inter-period optimization problem and hence it is solved using the same optimization technique as the inter-period optimization problem. On the other hand, SDP can accommodate a benefit function generated using any type of intra-period pre-commutation method.

This study attempts to solve the intra-period single reservoir mixed-use network optimization problem of the standard dynamic programming problem, which amounts to the term $N B_{t}(r)$ of the convex programming problem,

$$
\max _{r, S}\left(\sum_{t=1}^{T} N B_{t}\left(r_{t}\right)+V_{T+1}\left(s_{T+1}\right)\right)
$$

for some particular period, $t$. As already noted in the above, $N B_{t}(r)$ denotes this intra-period benefit function which includes both demanders and suppliers in a nodal catchment.

$\sum_{m} V_{m}^{T} s_{m}^{T}$ is also assumed to be an appropriately discounted piecewise linear, increasing, convex, end of horizon benefit storage function. It represents the value that would be achieved by the market if water is carried forward, beyond the current planning horizon.

\section{INTRA-PERIOD MULTI-NODAL CDDP PROCEDURE}

Instead of solving a whole series of sub-problems explicitly, the CDDP procedure for clearing the intra-period nodal market implicitly defines (marginal) benefit as a function of release, across the whole range of possible releases in the current period, by directly constructing marginal water value curves to compute the reservoir $d c r$. Note that this is equivalent to optimizing model (1) - (5) for all values of $r$ simultaneously, in a single pass. Arc bounds restrict the opportunities which may be exploited at connected nodes. The cumulative effect of these restrictions is maintained by a domain associated with each marginal value curve. We use the notation $f: S$ to denote function $f$ restricted to domain $S$.

We define the catchment optimization model used to determine the optimal net benefit function, $N B(r)$, as a function of net reservoir release, $r$. The $d c r$ is derived from $N B(r)$ as $\operatorname{dcr}(r)=N B^{\prime}(r)$. Here $r$ represents the net decrease in storage level and thus it can take negative or positive values.
Let $\mathrm{T}=(N=\{0,1, \ldots, n\}, A)$ be a tree with the reservoir at the root node 0 , and nodes, $i \in N$ indexed so that child nodes (further from the reservoir) have a larger index than their parents. Arc $(i, j) \in A$ has the usual water flow direction from node $i$ to node $j$.

The amount of inflow captured at node $i$ is denoted, $\bar{F}_{i} \leq f_{i} \leq \underline{F}_{i}$. However, in most general cases the lower bound of the tributary flows can be zero. A non-zero lower bound may be enforced in order to maintain a minimum in-stream flow requirement in a tributary flow path (arc). Tributary inflows not captured (if any) are assumed as a lost to the system. For full generality, $C F_{i}\left(f_{i}\right)$ denotes the convex cost function associated with the capture of this flow. This generality allows for supply participants who incur costs or who charge for the flow injection. This could also be water purchased from a different catchment (e.g., traded between water authorities) or a desalination plant (e.g., urban reservoir system). For a typical tributary flow $C F_{i}\left(f_{i}\right)=0$. We could model inflows which include upstream non-consumptive uses by allowing $C F_{i}$ to be negative. For example, $C F_{0}<0$ reflects non-consumptive benefits of upstream flow users directly above the reservoir.

Each non-root node $i$ has $C_{i}>0$ units of (potential) consumptive demand, with an associated concave benefit function $C B_{i}\left(c_{i}\right)$. Variable $0 \leq c_{i} \leq C_{i}$, represents the consumptive demand met at node $i$.

Variable $0 \leq d_{i} \leq E_{i}$ represents distributary flow from non-root node $i$ out of the system. The target flow is $E_{i}(\geq 0)$, with a convex cost function $C E_{i}\left(E_{i}-d_{i}\right)$ associated with missing the target. These distributary flows may be used to apply desired environmental flow requirements.

Nodes without distributary flow, tributary flow, and/or consumptive demand would have the corresponding bounds set to zero. We do not account for consumptive and distributary return flows and assume these flows are completely lost to the system. Each $\operatorname{arc}(i, j) \in A$ has an associated variable $x_{i j}$ representing downstream flow, within flow limits: $\underline{X}_{i j} \leq x_{i j} \leq \bar{X}_{i j}$. The lower bound may be negative for a syphon or if pumping or some other upstream transfer were available.

We refer to non-consumptive flow as flow on an arc which creates a benefit or cost to the system, such as hydropower production or pumping. The net benefit received from a non-consumptive arc flow of $x$ over $\operatorname{arc}(i, j)$ is denoted by $N C F_{i j}(x)$ (This is negative for costs incurred, for example, from pumping). We ignore issues such as power conversion functions and reservoir head effects in hydroelectric production.

The model data is pre-processed to simplify the procedure description. All cost and benefit functions at nodes are converted to marginal net demand functions. Here, these are described as defining marginal prices as a function of water supplied. But they could, equivalently, be expressed as defining net demand as a function of price. Specifically, for each node $i$, the pre-processing calculates the following net demand functions.

Tributary flows form net demand $c f_{i}:\left[-F_{i}^{\max }, 0\right]$ where $c f_{i}(f)=\frac{d}{d q} C F_{i}(-f)$.

Consumptive demands form net demand $c b_{i}:\left[0, C_{i}\right]$ where 
$c b_{i}(c)=\frac{d}{d q} C B_{i}(c)$

Distributary flows form net demand $c e_{i}:\left[0, E_{i}\right]$ where $c e_{i}(d)=\frac{d}{d q} C E_{i}\left(E_{i}-d\right)$.

By convention, arcs are directed away from the reservoir. The flow bounds for arc $j$ are adjusted to account for this, setting $L_{j}^{\prime}=L_{j}$ and $U_{j}^{\prime}=U_{j}$ if water flow is away from the reservoir, and $L_{j}^{\prime}=-U_{j}$ and $U_{j}^{\prime}=-L_{j}$ if water flow is directed towards the reservoir. Non-consumptive uses are similarly affected forming net demand function $n c f_{j}:\left[L_{j}^{\prime}, U_{j}^{\prime}\right]$ where $n c f_{j}(x)=\frac{d}{d x} N C B_{i}(x)$ for arcs with water flow away from the reservoir and $n c f_{j}(x)=-\frac{d}{d x} N C B_{i}(-x)$ otherwise.

The following procedure constructs the $d c r$ corresponding to $N B(r)$ from the previous model. In the procedure below, par(i) denotes the parent of node $i$.

Procedure Demand curve for release $(d c r)$ for single tree reservoir network

$n b_{i}:\left[a_{i}, b_{i}\right]$ is the nodal demand curve for flow, $n b_{i}=$

$0, a_{i}=0, b_{i}=0 \quad \forall i=0, \ldots, n$

For $i=n$ to 1 Step -1 do

$$
\begin{aligned}
& \mid \begin{array}{l}
n b_{i}:\left[a_{i}, b_{i}\right] \leftarrow\left[\begin{array}{c}
n b_{i}:\left[a_{i}, b_{i}\right]+{ }^{h} c b_{i}:\left[0, C_{i}\right]+{ }^{h} c e_{i}:\left[0, E_{i}\right] \\
+{ }^{h} c f_{i}:\left[-F_{i},-\bar{F}_{i}\right]
\end{array}\right] \\
\operatorname{set}(j, k)=(i, \operatorname{par}(i)) \text { or }(j, k)=(\operatorname{par}(i), i) \\
\overline{n b}_{l}:\left[\underline{X}_{j k}^{\prime}, \bar{X}_{j k}^{\prime}\right]=\text { truncate }\left(n b_{i}:\left[a_{i}, b_{i}\right],\left[\underline{X}_{j k}^{\prime}, \bar{X}_{j k}^{\prime}\right]\right) \\
/ / \text { Truncated to arc limits } \\
\widetilde{n b}_{l}:\left[\underline{X}_{j k}^{\prime}, \bar{X}_{j k}^{\prime}\right]=\left[\overline{n b}_{i}:\left[\underline{X}_{j k}^{\prime}, \bar{X}_{j k}^{\prime}\right]+{ }^{v} n c f_{i}:\left[\underline{X}_{j k}^{\prime}, \bar{X}_{j k}^{\prime}\right]\right] \\
n b_{p a r(i)}:\left[a_{p a r(i)}, b_{p a r(i)}\right] \leftarrow \\
{\left[\begin{array}{l}
\left.n b_{p a r(i)}:\left[a_{\text {par }(i)}, b_{p a r(i)}\right]+{ }^{h} \widetilde{n b}_{i}:\left[\underline{X}_{j k}^{\prime}, \bar{X}_{j k}^{\prime}\right]\right] \\
\text { Next } i / / \operatorname{Repeat~process~until~reach~node~} 0 .
\end{array}\right.}
\end{array} \\
& \text { Output:dcr } \leftarrow\left[n b_{0}:\left[a_{0}, b_{0}\right]+{ }^{h} c f_{0}:\left[-\underline{F}_{0},-\bar{F}_{0}\right]\right]
\end{aligned}
$$

The procedure starts from the node with the largest index (which must be a leaf node). A nodal net demand curve $(n b:[a, b])$ is formed by "horizontally adding" all tributary flows, distributary, and consumptive demands $(c f, c e, c b)$ at the node with any previously transferred demands (e.g., demand curve of a child node). This horizontal adding corresponds to ordering the net demands from all of these sources, from the most to the least valuable. The bounds $[a, b]$ give the feasible range of net supply that could be used to meet some or all of these net demands. The following gives details on horizontal adding. For future illustrations, we use the term "net nodal consumptive demand curve," $n c b$ to represent the horizontal addition of just $c f, c e$ and $c b$ at a node. This gives a notational simplicity to express the local water off-take at a particular node.

The net demand curve, $(n b:[a, b])$, is then transferred to the parent node by first truncating the domain using arc flow bounds $[\underline{X}, \bar{X}]$; then "vertically adding" any non-consumptive flow demand components $n c f:[\underline{X}, \bar{X}]$. It forms $(\widetilde{n b}: \tilde{S})$. The vertical adding corresponds to adjusting prices due to additional benefits or costs arising from the non-consumptive uses. The above defines a transferred net demand curve at the parent node. Thus, the parent node will have a net nodal consumptive demand curve and $(\widetilde{n b}: \tilde{S})$ coming from this child node. The process is repeated until we reach the reservoir (node 0 ).

To ensure feasibility the truncate procedure pads $n b_{i}:\left[a_{i}, b_{i}\right]$ if $\left[a_{i}, b_{i}\right]$ does not cover $\left[\underline{X}_{j k}^{\prime}, \bar{X}_{j k}^{\prime}\right]$. In particular, quantities higher than $b_{i}$ are given price zero while those below $a_{i}$ are given a high penalty value. These adjustments can be avoided by careful construction of the data. For simplicity, the proof the above procedure that constructs the required $d c r$ is excluded from this discussion (refer to [3] for the proof of the above procedure). The next section shows a numerical example of the above procedure applied to a single reservoir mixed-use nodal catchment.

\section{ILLUSTRATIVE EXAMPLE}

Let us use the following single reservoir river network with a set of hypothetical consumptive, and non-consumptive demands to illustrate the single reservoir nodal CDDP procedure (see Fig. 2 below). Solid triangle represents the reservoir. Solid circle is a consumptive node in the network. A non-consumptive use may happen in an arc. For example, the turbine symbol on arc $(0,1)$ in the following figure shows a hydro-power generator.

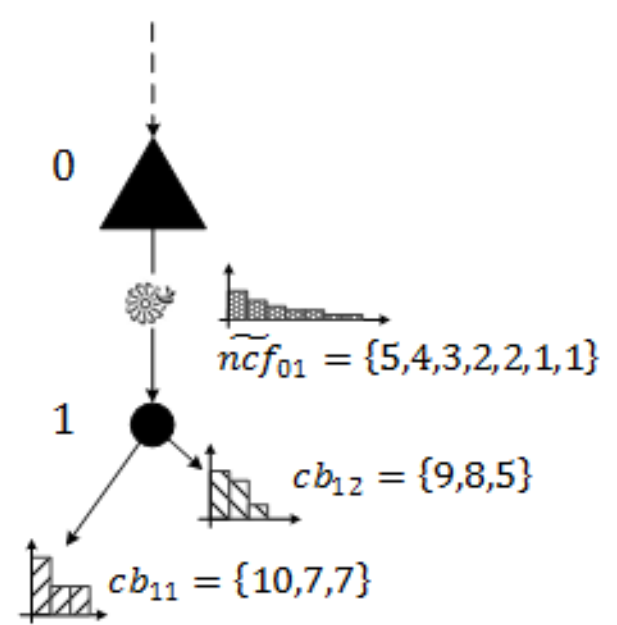

Fig. 2. Consumptive $\&$ non-consumptive demands across network.

\section{A. Constructing the Nodal Demand Curve for Consumptive Use}

Consider a single node located upstream from the reservoir, as in Fig. 3 (which shows detail from Fig. 2). The procedure then constructs the nodal demand curve for water $n b($. incrementally, by horizontal addition: $n b_{1}: S_{1} \leftarrow$ $\left[c b_{11}:[0,3]+{ }^{h} c b_{12}:[0,3]\right]$. This is equivalent to sorting all increments of consumptive demand by decreasing incremental value.

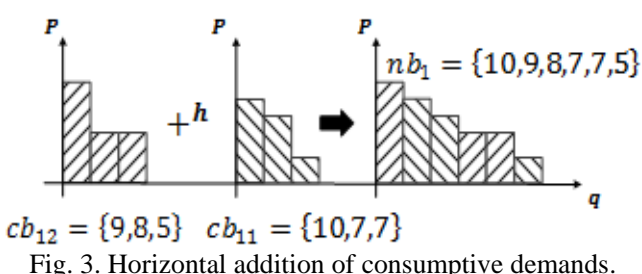




\section{B. Processing Non-consumptive Use}

The water flow direction on arc $(0,1)$ is away from the reservoir. The flow limits are on such arcs will imply non-negative upper/lower limits on the net demand curve passed back through the tree, towards the reservoir. And non-consumptive uses on such arcs will add positive increments to the prices in those demand curves, representing the additional non-consumptive benefit from flow away from the reservoir, in this case. Fig. 4 illustrates the formation of $n b_{0} \leftarrow \widetilde{n b_{1}}:[0,6]$.

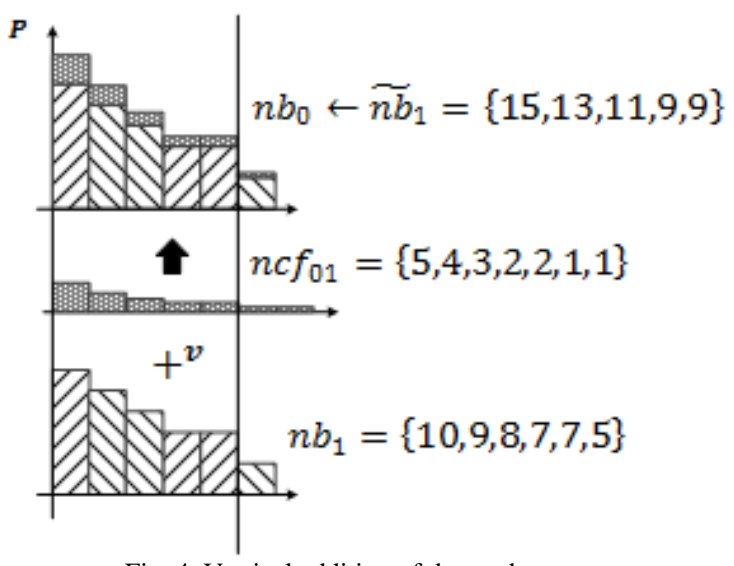

Fig. 4. Vertical addition of demand curves.

Finally, we reach node 0, where we obtain the $d c r$ for the reservoir: $d c r \leftarrow n b_{0}$. Suppose, the reservoir receives 1 unit of inflows priced at zero value shown by dashed arrow. The procedure now allocates the supply unit to the highest bid of 15. So, the net demand curve for release is net $d c r=$ $\{13,11,9,9\}$.

\section{CONCLUSIONS}

A nodal catchment model can be applied to optimise usage over time, or to clear an inter-temporal market for any catchment with one reservoir and a tree configuration. To solve the model, we proposed a simple and efficient two-level application of CDDP.

First, a multi-nodal deterministic CDDP, presented here, constructs aggregate demand curves for release in each period. A stochastic CDDP could construct aggregate demand curves for storage in the single reservoir for each period. For example, see [8]. The complexity of the intra-period network can readily be increased by adding upstream and downstream nodes, and physical links, while still maintaining efficient computation.

Increasing the number of long term storage reservoirs will increase the complexity of the CDDP procedure as a result of "curse of dimensionality". But [8] describe several two reservoir implementations of stochastic CDDP, while [7] describe development work on a multi-reservoir generalisation of the stochastic inter-period procedure.

Further extensions such as intra-period and inter-period stochastic CDDP. The two-reservoir CDDP and multi-reservoir CDDP models could be studied, but probably with only mixed success ([3] and [7]). Dimensionality issues may preclude development in some directions, and the most efficient implementation approach is unclear in others. We are motivated, though, to explore avenues to use the stochastic CDDP to model/simulate how a market responds to stochastic inflows influenced by el niňo-la niňa weather patterns.

\section{ACKNOWLEDGEMENT}

I will be forever grateful to my doctoral study supervisors Professor Grant Read and Dr. Shane Dye attached to University of Canterbury, Christchurch, New Zealand for their guidance, kindness, friendship, and continuous support throughout my doctoral studies.

\section{REFERENCES}

[1] S. Dye, E. G. Read, R. A. Read, and S. R. Starkey, "Easy implementations of generalised stochastic CDDP models for market simulation studies," Cigré International Workshop on Hydro Scheduling in Competitive Markets, Bergen, Norway, 2012.

[2] S. R. Starkey, "Water allocation under uncertainty - Potential gains from optimisation and market mechanisms," Ph.D. dissertation, Department of Management, Marketing and Entrepreneurship, University of Canterbury, 2014.

[3] I. Mahakalanda, "An integrated water-electricity market design for multi-reservoir, mixed catchments," Ph.D. dissertation, Department of Management, Marketing and Entrepreneurship, University of Canterbury, 2016.

[4] K. A. McCabe, S. J. Rassenti, and V. L. Smith, "Smart computer-assisted markets," Science (New York, N.Y.), vol. 254, no. 5031, pp. 534-538, 1991

[5] J. J. Murphy, A. Dinar, R. E. Howitt, S. J. Rassenti, and V. L. Smith, "The design of smart water market institutions using laboratory experiments," Environmental and Resource Economics, vol. 17, no. 4, pp. 375-394, 2000

[6] J. Raffensperger, "Matching users' rights to available groundwater," Ecological Economics, vol. 70, no. 6, pp 1041-1050, 2011.

[7] E. G. Read and M. Hindsberger, "Constructive dual DP for reservoir optimization," Handbook of Networks in Power Systems I, 2010, pp 3-32.

[8] T. J. Scott and E. G. Read, "Modelling hydro reservoir operation in a deregulated electricity market," International Transactions in Operational Research, vol. 3, no. 3, pp. 243-253, 1996.

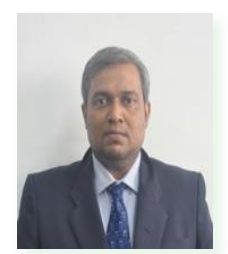

Indra Mahakalanda received the $\mathrm{Ph}$. D. degree in management science from University of Canterbury, New Zealand in 2016. He is currently the head, the Department of Decision Sciences, Faculty of Business, University of Moratuwa, Sri Lanka. 